\title{
Screening for Drought Tolerance in Thirty Three Taro Cultivars
}

\author{
José F.T. GANANÇA ${ }^{1,2, *}$, José G.R. FREITAS ${ }^{1}$, Humberto G.M. \\ NÓBREGA ${ }^{1}$, Vanessa RODRIGUES ${ }^{1}$, Gonçalo ANTUNES ${ }^{1}$, \\ Carla S.S. GOUVEIA ${ }^{1}$, Miguel RODRIGUES ${ }^{3}$, Hana CHAÏR ${ }^{4,5}$, \\ Miguel Â.A. PINHEIRO de CARVALHO ${ }^{1,2}$, Vincent LEBOT $^{6}$ \\ ${ }^{1}$ University of Madeira, ISOPlexis Genebank, 9020-105 Funchal, Portugal; jofitei@staff.uma.pt ("corresponding author); \\ gregoryus@hotmail.com; humberto_nobrega@yahoo.com;vannyisa@hotmail.com; \\ goncalonuno80@gmail.com; carlassgouveia@hotmail.com; quercus@staff.uma.pt \\ ${ }^{2}$ Institute for Mediterranean Environmental and Agrarian Sciences (ICAAM), University of Évora, Évora, Portugal \\ ${ }^{3}$ Regional Directorate for Agriculture and Rural Development (DRADR),Funchal, Portugal; miguelrodrigues@gov-madeira.pt \\ ${ }^{4}$ CIRAD, UMR AGAP, F-34398 Montpellier, France; hana.chair@cirad.fr \\ ${ }^{5}$ AGAP, Univ Montpellier, CIRAD, INRA, INRIA, Montpellier Sup Agro, Montpellier, France \\ ${ }^{6}$ CIRAD, UMR AGAP, PO Box 946, Port-Vila, Vanuatu; lebot@vanuatu.com.vu
}

\begin{abstract}
Taro [Colocasia esculenta (L.) Schott] is a root crop which is an important staple food in many regions of the world, producing 10.5 million tonnes on 1.4 million hectares a year. The crop is cultivated in wet (rain fed) or irrigated conditions, requiring on average $2,500 \mathrm{~mm}$ water per year, and in many countries it is cultivated in flooded plots. It is estimated that taro production could decrease by $40 \%$ as a result of the increase in drought and other severe events. In this work, thirty three accessions, including local cultivars, selected and hybrid lines were submitted to long duration drought stress and screened for tolerance. Twelve physiological, morphological and agronomic traits were measured at harvest, and subject to multivariate analysis. Stress indices, Water Use Efficiency and Factorial Analysis were useful for discriminating accessions regarding drought tolerance and yield stability, and drought tolerant and susceptible cultivars were identified. Our results confirm that different taro cultivars have different drought avoidance and tolerance strategies to cope with water scarcity. Better yield performers minimised biomass and canopy loss, while tolerance was observed in cultivars that presented low potential yield, but efficiently transferred resources to enhance corm formation. Among the 33 accessions, two local cultivars showed high yield stability and could be considered as suitable parents for breeding programs, while two others are well adapted to drought, but with overall low yield potential.
\end{abstract}

Keywords: abiotic stress, multicriteria indices, plant water use, taro breeding, yield stability

\section{Introduction}

Taro [Colocasia esculenta (L.) Schott] is an underutilized root crop, originating from the Southeast Asia (Rao et al., 2010; Mabhaudhi and Modi, 2015), which is an important staple food in many regions of the world, producing 10.5 million tonnes on 1.4 million hectares, with an average yield of $7.5 \mathrm{t} / \mathrm{ha}$ (FAOSTAT, 2013). It is grown through the Pacific Islands, Asia, Africa, Europe and the Caribbean Islands, where thousands of cultivars adapted to different agro-ecological conditions are maintained by local farmers.
The crop is cultivated under wet (rain fed) or irrigated conditions, on flooded plots, or on dry fields requiring on average 2,500 $\mathrm{mm}$ rainfall per year, to obtain optimal yields (Onwueme, 1999). The taro genetic diversity outside Southeast Asia is considered narrow, which makes the crop vulnerable to a range of damaging biotic factors (Rao et al., 2010). Similarly, it makes taro vulnerable to abiotic constraints, namely increasing frequency and intensity of droughts, resulting from ongoing climate changes affecting the main producing countries (Wairiu et al., 2012).

Under the current scenario, it is estimated that taro production during the next 30 years could decrease by $40 \%$ as a result of the increase in drought and other severe weather events (Wairiu et al., 2012). Little is known about the crop performance under drought conditions. Several 
66

morphological, agronomic, yield and physiological parameters have been used to assess varietal performance of taro cultivars with regard to drought tolerance (Sivan, 1995; Bussel and Bonin, 1998; Manyatsi et al., 2011; Mabhaudhi et al., 2013; El-Zohiri and Abd El-Aal, 2014; Mabhaudhi and Modi, 2015). Unfortunately, no information is available regarding the genetic control of drought tolerance in taro, hindering the possibility of fast screening of the crop towards drought tolerance.

Recently, Mabhaudhi et al. (2014) used the AquaCrop model to simulate yield responses to water supply of a South African eddoe type taro landrace. However, the model still showed some limitations in simulating taro growth under moisture stress, and more information is required to develop a comprehensive drought stress model. Moreover, in this crop its difficult to replicate or to maintain stress conditions under controlled environments (Ganança et al., 2015). A major issue to be addressed is still, therefore, the establishment of the crop drought stress conditions, as well as the detection of traits that can specifically discriminate the variation of plant performance under drought. However, from a breeders perspective, yield stability under dry conditions is the main objective (Ganjeali et al., 2011). In fact, genotypes that have high yield under both stressed and non-stressed conditions could be considered drought resistant (Blum, 2005), and plant screening should be conducted based on the comparison of fitness and high performance under these conditions (Ganjeali et al., 2011). Several indices were developed to evaluate crop yield stability under stress conditions. Stress Tolerance Index (STI) (Fernandez, 1992), and Stress Susceptibility Index (SSI) (Fisher and Maurer, 1978) are two of the most frequently used (Ganjeali et al., 2011). These indices were applied to evaluate yield stability under drought stress conditions in mungbean (Fernandez, 1992), chickpea (Ganjeali et al., 2011), and wheat (Farshadfar et al., 2013).

To date, research attempts in taro have focused on describing the effects of drought on morphological and physiological traits. Crop productivity expressed as Harvest Index (HI) has been used to assess and compare a traditional Samoan cultivar and an improved drought resistant cultivar (Bussel and Bonin, 1998), as well as South African cultivars (Mabhaudhi et al., 2015) for their suitability to growth under stress conditions. To the best of our knowledge, no attempts to identify taro genotypes suitable for breeding towards drought tolerance trough evaluations of yield stability under conditions of soil moisture deficit have been reported. The objectives of this study were to screen a large number of taro cultivars for drought tolerance using easy to record morpho-agronomic parameters, and evaluate yield stability as a measure of taro drought tolerance.

\section{Materials and Methods}

\section{Plant materials and culture}

Evaluation of taro cultivars for drought resistance was conducted in open greenhouses in the Preces experimental station, Câmara de Lobos, Madeira, Portugal ( $32^{\circ} 39^{\prime} \mathrm{N}$; $16^{\circ} 58^{\prime} \mathrm{W}$ ) during a full plant growth cycle (from June 2013 to July 2014).
Thirty three taro accessions (Table 1), including 14 breeders lines or elite cultivars from TANSAO core collection provided by the Secretariat of the Pacific Community (SPC, Fiji), and 19 cultivars from Europe (ten from Madeira, six from Canary Islands, two from Azores and one from Cyprus) were studied. Before the drought assay (2012-2013), plants of all cultivars were multiplied, acclimatized and maintained in the experimental greenhouses. Ploidy levels of the studied varieties (whenever available) were obtained from Traoré (2013) and Kreike et al. (2004).

\section{Experimental design}

In June 2013, a total of 660 plants were established in individual $30 \times 30 \mathrm{~cm}$ pots filled with $15 \mathrm{~kg}$ of dried soil. The pots were arranged in rows spaced $90 \mathrm{~cm}$ apart, with 30 $\mathrm{cm}$ in row separation. Each pot was treated as a single soilplant system and all were laid out in a randomized complete block design. Twenty corms heads per cultivar at the same development stage and size were collected. Ten plants per cultivar were used as control (fully watered) and ten plants were submitted to stress conditions (experimental variant). The experimental design was a factorial experiment with two factors: irrigation level and cultivar.

\section{Experimental conditions}

Before potting, soil in pots was amended with compost in a 3:1 proportion, sampled and analysed for its physical and edaphic properties, including major mineral nutrients content (data not shown). The soil was classified as Sandy Clay Loam (USDA taxonomic system). Soil $\mathrm{pH}$ (water) was 5.7 and organic mater content was $14.65 \%$. The soil water characteristics hydraulic properties calculator (http://hydrolab.arsusda.gov/soilwater/Index.htm) was used to calculate soil field capacity (37.1\%), permanent wilting point (22.6\%), and saturation (58.4\%). Following soil analyses, fertilizer application during the experiment was not deemed necessary. Manual pot weeding was done regularly.

Irrigation regimes were defined based on a test pilot were evapotranspiration (ET) at field capacity (FC) was monitored during four weeks, in the acclimation year (three weeks in July-August and one week in November) in the real pot plant-soil system, with six fully developed taro plants. Evapotranspiration (ET) was measured daily by weighting the pot, and compensating for water loss (Dwyer, 1987) during each week of the pilot study. Under greenhouse conditions, ET averaged $10.7 \pm 3.5 \mathrm{~mm}^{-1}$ day $^{-1}$ (ranging from 5.6 to $13.7 \mathrm{~mm} \cdot \mathrm{day}^{-1}$ ). This value was subsequently used to define water irrigation regimes in the actual experiment. After planting, all pots were brought to full FC and equally irrigated $\left(10.7 \mathrm{~mm} \cdot\right.$ day $\left.^{-1}\right)$ until desired experimental conditions were met. Drought conditions were imposed from October 2013 until the harvest 298 days later (July 2014). Control plants (100\% water requirement) received in total $3,373.4 \mathrm{~mm}$.pot ${ }^{-1}$ at rate of $10.7 \mathrm{~mm}$. day $^{-1}$. The stress variant (53\% water requirement) was provided in total with $1,812.8 \mathrm{~mm} \cdot$ pot $^{-1}$ at rate of 5.7 mm.day ${ }^{-1}$. Irrigation was delivered daily using a drip irrigation system with an average discharge rate per dripper of $2.9 \mathrm{~L} \cdot \mathrm{h}^{-1}$. 
Table 1. ISOPlexis Genebank accession, designation and geographical origin, and ploidy levels of the cultivars used in the drought stress assay

\begin{tabular}{|c|c|c|c|}
\hline Accession & $\begin{array}{c}\text { Cultivar } \\
\text { name }\end{array}$ & Origin & $\begin{array}{l}\text { Ploidy } \\
\text { levels }\end{array}$ \\
\hline 2056 & Listado & Canary Islands - La Palma & n.d. \\
\hline 2057 & Colorado & Canary Islands - La Gomera & n.d. \\
\hline 2058 & Morado & Canary Islands - La Palma & n.d. \\
\hline 2060 & Barranquera & Canary Islands - Gran Canaria & n.d. \\
\hline 2061 & Blanco Saucero & Canary Islands - La Palma & n.d. \\
\hline 2062 & Barranquera de los Sauces & Canary Islands - La Palma & n.d. \\
\hline 2183 & 190/05 Branco & Azores Islands - Terceira Island & n.d. \\
\hline 2184 & Vermelho & Azores Islands - Terceira Island & n.d. \\
\hline 2186 & Kolokasi & Cyprus & n.d. \\
\hline 2207 & Roxo & Madeira Island & $3 x$ \\
\hline 2208 & Branco & Madeira Island & n.d. \\
\hline 2209 & Branco & Madeira Island & n.d. \\
\hline 2210 & Roxo & Madeira Island & $3 x$ \\
\hline 2211 & Branco & Madeira Island & n.d. \\
\hline 2212 & Roxo & Madeira Island & n.d. \\
\hline 2213 & Branco & Madeira Island & n.d. \\
\hline 2214 & Roxo & Madeira Island & n.d. \\
\hline 2215 & Roxo & Madeira Island & n.d. \\
\hline 2216 & Branco & Madeira Island & $3 x$ \\
\hline 2232 & PE×PH 15-6 BL/HW/08 & SPC, Fiji & $2 x$ \\
\hline 2233 & C3-12 BL/PNG/10 & SPC, Fiji & $2 x$ \\
\hline 2234 & $\mathrm{C} 3-22 \mathrm{BL} / \mathrm{PNG} / 11$ & SPC, Fiji & $2 x$ \\
\hline 2235 & Samoa43 BL/SM/43 & SPC, Fiji & $2 x$ \\
\hline 2236 & Lepa BL/SM/149 & SPC, Fiji & $2 x$ \\
\hline 2237 & Ngerruch CE/PAL/10 & SPC, Fiji & n.d. \\
\hline 2238 & Karang CE/MAL/08 & SPC, Fiji & n.d. \\
\hline 2239 & Karang CE/MAL/10 & SPC, Fiji & n.d. \\
\hline 2240 & Lebak CE/Ind/16 & SPC, Fiji & $2 x$ \\
\hline 2241 & Manokwari CE/Ind/31 & SPC, Fiji & $2 x$ \\
\hline 2242 & Srisamrong CE/THA/07 & SPC, Fiji & $2 x$ \\
\hline 2244 & Boklua CE/THA/24 & SPC, Fiji & $2 x$ \\
\hline 2245 & Wasehasuba-Imo CA/JP/02 & SPC, Fiji & $2 x$ \\
\hline 2246 & Takenoko-Imo CA/JP/08 & SPC, Fiji & n.d. \\
\hline
\end{tabular}

\section{Data collection and statistical analysis}

For each cultivar, seven morphological, one physiological and three agronomic traits were measured at harvest on five randomly selected plants per treatment (Tables 2, 3, 4, 5). Chlorophyll content index was measured using a CCM-200 plus chlorophyll content meter from Opti-Sciences (USA). Leaf Area (LA) was calculated as a function of leaf width and length according to Manyatsi $e t$ al. (2011). Water Use Efficiency (WUE) was calculated as the ratio of total plant biomass to total water used per pot, and expressed in g.m. $\mathrm{m}^{-3}$. Harvest Index (HI) was adapted from Mabhaudhi et al. (2013). Stress Tolerance Index (STI) and Stress Susceptibility Index (SSI) were adapted from Ganjeali et al. (2011) and Farshadfar et al. (2013), respectively, using corm biomass as a measure of yield. Statistical analysis, with variance analysis, correlations and factorial analysis, were performed using SPSS for Windows, version 22.

\section{Results}

\section{Growth traits}

Under controlled conditions, leaf number (LN), plant height $(\mathrm{PH})$ and leaf area (LA), (Tables 2, 3, 4), were significantly higher than under stress $(\mathrm{p} \leq 0.001)$. Also, the three parameters differed significantly among cultivars $(\mathrm{p} \leq$ 0.001 ). A significant treatment $\times$ genotype interaction was detected for PH and LA ( $p \leq 0.001)$, but not to LN ( $p \leq$ $0.05)$. PH and LN varied significantly between stress and non stress conditions, but we can not confirm that observed $\mathrm{LN}$ variation between the treatments could be attributed to drought conditions. Mean LN under stress was on average $83.20 \%$ of the control plants, and the majority of cultivars (27) had equal or less leaves under stress conditions than under control. All cultivars presented a decreased plant height under stress, ranging between -11.62 and $-66.36 \%$ of the total height. Mean PH under stress was on average 
68

$62.34 \%$ of the control, and LA under stress was on average $48.66 \%$ of the control plants.

These results indicate that $\mathrm{PH}$ and LA could be used to discriminate cultivars with regard to drought tolerance, while $\mathrm{LN}$ appeared to be an unusable indicator.

\section{Chlorophyll Content Index (CCI)}

Chlorophyll Content Index (CCI) showed great variability among the cultivars (Tables 3 and 4). Under stress conditions, 22 cultivars showed higher CCI, while 11 had lower CCI. Mean CCI was significantly higher ( $\mathrm{p} \leq$ $0.001)$ under stress than under control (22.16\% higher). Performance among cultivars differed significantly in relation to CCI $(\mathrm{p} \leq 0.001)$. Significant treatment $\times$ genotype interaction $(\leq 0.001)$ was observed, revealing that, at least in some cultivars, CCI varied between stress and non stress conditions.

\section{Water Use Efficiency (WUE)}

WUE was used to assess taro's capacity to face water scarcity (Table 4). On average, WUE decreased 35.42\% under stress, with cultivar 2061 showing the best performance. Mean WUE was significantly higher under control than under stress $(\mathrm{p} \leq 0.001)$. WUE differed significantly among the cultivars, and a significant treatment $\times$ genotype interaction $(\mathrm{p} \leq 0.001)$ was detected, showing a significant parameter variation under stress in some genotypes.

Table 2. Data of morphological, physiological, and agronomic parameters obtained under control conditions

\begin{tabular}{|c|c|c|c|c|c|c|c|c|c|}
\hline Accession & CCI & $\mathrm{LN}$ & $\begin{array}{l}\mathrm{PH} \\
(\mathrm{cm})\end{array}$ & $\begin{array}{c}\text { LA } \\
\left(\mathrm{cm}^{2}\right)\end{array}$ & $\begin{array}{c}\text { TPB } \\
(\mathrm{g})\end{array}$ & $\begin{array}{c}\text { FAGW } \\
(\mathrm{g})\end{array}$ & $\begin{array}{c}\text { DAGW } \\
(\mathrm{g})\end{array}$ & $\begin{array}{c}\text { DCW } \\
(\mathrm{g})\end{array}$ & $\begin{array}{c}\text { FCW } \\
(\mathrm{g})\end{array}$ \\
\hline 2056 & 39.57 & 2.20 & 48.86 & 325.76 & 347.00 & 83.43 & 8.28 & 15.27 & 185.43 \\
\hline 2057 & 30.98 & 1.80 & 57.06 & 705.95 & 568.00 & 175.60 & 15.18 & 72.94 & 335.56 \\
\hline 2058 & 22.67 & 2.00 & 67.78 & 576.16 & 836.00 & 171.89 & 15.74 & 167.57 & 610.27 \\
\hline 2060 & 32.98 & 1.20 & 52.50 & 483.49 & 744.00 & 100.04 & 11.99 & 102.61 & 442.36 \\
\hline 2061 & 25.77 & 2.60 & 66.76 & 668.20 & 986.00 & 293.25 & 37.29 & 177.40 & 621.87 \\
\hline 2062 & 35.98 & 1.80 & 53.96 & 851.04 & 473.00 & 154.53 & 15.54 & 33.08 & 230.53 \\
\hline 2183 & 27.99 & 1.60 & 49.50 & 375.62 & 634.00 & 119.92 & 7.70 & 73.17 & 444.76 \\
\hline 2184 & 21.47 & 1.40 & 58.06 & 662.23 & 600.00 & 139.42 & 9.33 & 86.46 & 407.18 \\
\hline 2186 & 22.71 & 1.80 & 54.04 & 485.60 & 569.00 & 118.01 & 15.57 & 70.37 & 381.71 \\
\hline 2207 & 32.16 & 2.00 & 56.84 & 491.63 & 592.00 & 113.64 & 8.42 & 64.03 & 381.89 \\
\hline 2208 & 30.97 & 1.40 & 48.76 & 682.28 & 682.00 & 134.86 & 23.09 & 80.31 & 493.08 \\
\hline 2209 & 24.03 & 2.00 & 58.12 & 837.46 & 881.00 & 194.53 & 17.05 & 117.44 & 585.78 \\
\hline 2210 & 2.20 & 2.20 & 41.08 & 276.71 & 314.00 & 76.43 & 9.13 & 40.59 & 191.59 \\
\hline 2211 & 29.07 & 2.20 & 67.90 & 990.61 & $1,788.00$ & 730.11 & 65.43 & 201.09 & 756.79 \\
\hline 2212 & 41.59 & 2.20 & 39.10 & 219.84 & 205.00 & 29.78 & 3.81 & 32.18 & 149.96 \\
\hline 2213 & 29.45 & 2.00 & 63.66 & $1,093.28$ & 967.00 & 258.68 & 27.21 & 112.11 & 594.49 \\
\hline 2214 & 24.26 & 2.20 & 56.96 & 480.67 & 665.00 & 155.09 & 28.16 & 112.93 & 414.60 \\
\hline 2215 & 49.75 & 2.20 & 43.94 & 220.15 & 244.00 & 52.04 & 5.37 & 34.56 & 143.52 \\
\hline 2216 & 41.17 & 2.00 & 82.10 & $1,883.19$ & $1,728.00$ & 618.50 & 47.22 & 112.51 & 770.55 \\
\hline 2232 & 22.65 & 1.60 & 60.98 & 378.03 & 304.00 & 39.04 & 11.13 & 40.30 & 175.27 \\
\hline 2233 & 28.33 & 2.50 & 63.58 & 446.57 & 403.75 & 144.13 & 14.75 & 61.68 & 175.43 \\
\hline 2234 & 28.96 & 2.60 & 55.04 & 298.74 & 200.00 & 64.18 & 9.27 & 19.61 & 63.97 \\
\hline 2235 & 27.24 & 3.00 & 61.80 & 355.17 & 295.00 & 160.64 & 11.48 & 20.05 & 105.17 \\
\hline 2236 & 22.27 & 2.60 & 45.06 & 157.05 & 261.00 & 49.90 & 10.83 & 54.97 & 177.14 \\
\hline 2237 & 33.73 & 2.60 & 63.92 & 588.16 & 496.00 & 219.11 & 18.80 & 42.49 & 178.89 \\
\hline 2238 & 32.29 & 2.40 & 44.76 & 223.95 & 351.00 & 76.99 & 8.59 & 48.28 & 214.49 \\
\hline 2239 & 17.20 & 2.80 & 43.74 & 307.40 & 410.00 & 133.60 & 12.37 & 39.84 & 199.60 \\
\hline 2240 & 24.53 & 2.20 & 43.04 & 299.39 & 560.00 & 251.49 & 21.68 & 39.10 & 189.96 \\
\hline 2241 & 29.30 & 2.80 & 62.18 & 520.53 & 583.00 & 304.70 & 20.71 & 63.53 & 207.46 \\
\hline 2242 & 41.91 & 3.40 & 35.86 & 215.08 & 115.00 & 48.47 & 5.42 & 12.17 & 74.85 \\
\hline 2244 & 17.58 & 2.00 & 40.18 & 146.86 & 199.00 & 22.23 & 3.66 & 31.61 & 136.14 \\
\hline 2245 & 55.17 & 2.80 & 35.66 & 230.84 & 279.00 & 69.92 & 8.04 & 32.75 & 163.83 \\
\hline 2246 & 49.71 & 3.00 & 31.64 & 54.23 & 49.00 & 11.69 & 2.13 & 8.19 & 24.00 \\
\hline Mean & $31.35 \mathrm{ab}$ & $2.22 \mathrm{ab}$ & $53.16 \mathrm{ab}$ & $500.97 \mathrm{ab}$ & $555.42 \mathrm{ab}$ & 161.09ab & 16.07ab & $67.31 \mathrm{ab}$ & $309.94 \mathrm{ab}$ \\
\hline S.E. & 1.62 & 0.09 & 1.98 & 61.30 & 68.28 & 26.72 & 2.29 & 8.40 & 35.81 \\
\hline Min & 17.20 & 1.20 & 31.64 & 54.23 & 49.00 & 11.69 & 2.13 & 8.19 & 24.00 \\
\hline Max & 55.17 & 3.40 & 82.10 & $1,883.19$ & $1,788.00$ & 730.11 & 65.43 & 201.09 & 770.55 \\
\hline
\end{tabular}

CCI - Chlorophyll Content Index; LN - Leaf Number; PH - Plant Height; LA - Leaf Area (Leaf Width $\times$ Leaf Length $\times 0,85$ ); TPB - Total Plant Biomass; FAGW Fresh Above Ground Weight; DAGW - Dry Above Ground Weight; DCW - Dry Corm Weight; FCW - Fresh Corm Weight. a - Significant differences between accessions (ANOVA, $\mathrm{p} \leq 0.05$ )

$\mathrm{b}$ - Significant differences between stress and non stress conditions (ANOVA, $\mathrm{p} \leq 0.05$ ) 
Table 3. Data of morphological, physiological, and agronomic parameters obtained under drought conditions

\begin{tabular}{|c|c|c|c|c|c|c|c|c|c|}
\hline Accession & $\mathrm{CCI}$ & $\mathrm{LN}$ & $\mathrm{PH}(\mathrm{cm})$ & $\mathrm{LA}\left(\mathrm{cm}^{2}\right)$ & TPB (g) & FAGW (g) & DAGW (g) & $\overline{D C W}(\mathrm{~g})$ & $\overline{F C W}(\mathrm{~g})$ \\
\hline 2056 & 22.83 & 1.80 & 40.18 & 305.64 & 330.00 & 54.38 & 6.94 & 44.71 & 229.41 \\
\hline 2057 & 32.83 & 1.40 & 24.76 & 130.37 & 104.00 & 18.80 & 2.02 & 11.52 & 73.55 \\
\hline 2058 & 72.22 & 1.20 & 31.28 & 262.28 & 331.00 & 33.46 & 3.99 & 25.77 & 158.62 \\
\hline 2060 & 19.09 & 1.20 & 36.82 & 431.58 & 296.00 & 52.44 & 7.32 & 14.36 & 77.06 \\
\hline 2061 & 37.77 & 1.40 & 55.46 & 567.56 & 565.00 & 157.25 & 24.39 & 87.73 & 376.98 \\
\hline 2062 & 75.29 & 2.00 & 25.80 & 201.72 & 85.00 & 44.14 & 4.81 & 3.96 & 27.39 \\
\hline 2183 & 39.69 & 2.00 & 32.24 & 142.59 & 223.80 & 28.28 & 3.95 & 16.86 & 137.91 \\
\hline 2184 & 32.51 & 2.40 & 29.62 & 257.15 & 115.00 & 38.14 & 4.93 & 7.49 & 48.90 \\
\hline 2186 & 44.73 & 1.80 & 33.30 & 225.69 & 150.00 & 47.44 & 5.39 & 20.21 & 103.47 \\
\hline 2207 & 21.67 & 2.60 & 40.42 & 379.87 & 306.00 & 93.35 & 9.79 & 33.59 & 145.65 \\
\hline 2208 & 33.11 & 1.40 & 40.96 & 346.63 & 108.00 & 53.76 & 6.02 & 6.25 & 34.58 \\
\hline 2209 & 47.81 & 1.80 & 29.62 & 290.70 & 123.00 & 36.52 & 3.72 & 9.74 & 70.63 \\
\hline 2210 & 69.35 & 1.60 & 27.54 & 116.71 & 105.00 & 19.95 & 2.67 & 15.85 & 57.96 \\
\hline 2211 & 58.81 & 1.60 & 33.70 & 400.85 & 276.00 & 68.36 & 9.42 & 25.57 & 157.27 \\
\hline 2212 & 19.90 & 1.80 & 29.98 & 222.80 & 149.00 & 43.20 & 3.61 & 22.28 & 85.34 \\
\hline 2213 & 46.30 & 1.60 & 37.74 & 427.27 & 244.00 & 60.56 & 6.38 & 27.37 & 157.22 \\
\hline 2214 & 58.53 & 1.80 & 34.24 & 174.69 & 259.00 & 31.50 & 3.07 & 50.46 & 190.60 \\
\hline 2215 & 35.22 & 2.20 & 30.44 & 160.67 & 139.00 & 32.52 & 3.48 & 23.18 & 91.56 \\
\hline 2216 & 29.11 & 2.20 & 46.56 & 861.63 & 451.00 & 196.96 & 19.02 & 29.24 & 200.13 \\
\hline 2232 & 24.25 & 1.25 & 33.48 & 157.14 & 51.25 & 18.51 & 2.48 & 4.15 & 18.13 \\
\hline 2233 & 54.08 & 1.50 & 44.50 & 233.52 & 179.17 & 59.54 & 4.34 & 34.15 & 131.46 \\
\hline 2234 & 29.68 & 1.60 & 38.12 & 237.24 & 214.00 & 52.41 & 6.39 & 40.60 & 160.94 \\
\hline 2235 & 21.13 & 1.67 & 22.40 & 85.69 & 18.33 & 9.25 & 0.97 & 0.54 & 2.94 \\
\hline 2236 & 33.14 & 2.50 & 34.15 & 124.07 & 124.00 & 12.39 & 2.32 & 22.10 & 67.57 \\
\hline 2237 & 42.27 & 1.00 & 21.50 & 88.82 & 15.00 & 9.06 & 1.11 & 0.48 & 3.19 \\
\hline 2238 & 42.91 & 2.20 & 34.92 & 199.34 & 126.00 & 40.001 & 4.61 & 13.11 & 58.14 \\
\hline 2239 & 34.08 & 2.80 & 31.74 & 153.72 & 292.00 & 91.20 & 11.25 & 31.65 & 159.95 \\
\hline 2240 & 53.08 & 2.40 & 38.04 & 246.89 & 264.00 & 77.68 & 9.86 & 29.40 & 136.43 \\
\hline 2241 & 40.13 & 2.25 & 31.20 & 179.10 & 273.00 & 29.10 & 3.28 & 29.14 & 113.22 \\
\hline 2242 & 24.71 & 2.00 & 29.30 & 110.52 & 139.00 & 12.91 & 2.50 & 34.61 & 108.84 \\
\hline 2244 & 12.93 & 1.60 & 30.30 & 140.09 & 171.00 & 26.72 & 3.55 & 26.77 & 115.71 \\
\hline 2245 & 41.76 & 2.25 & 19.80 & 103.33 & 98.75 & 17.54 & 2.72 & 14.72 & 62.75 \\
\hline 2246 & 12.96 & 2.00 & 23.58 & 102.48 & 31.00 & 13.48 & 2.18 & 2.84 & 9.56 \\
\hline Mean & $38.30 \mathrm{c}$ & 1.84 & $33.14 \mathrm{c}$ & $244.49 \mathrm{c}$ & $192.62 \mathrm{c}$ & $47.90 \mathrm{c}$ & $5.71 \mathrm{c}$ & $23.04 \mathrm{c}$ & $108.27 \mathrm{c}$ \\
\hline S.E. & 2.84 & 0.77 & 1.31 & 28.02 & 21.43 & 7.03 & 0.85 & 3.02 & 13.32 \\
\hline Min & 12.93 & 1.00 & 19.80 & 85.69 & 15.00 & 9.06 & 0.97 & 0.48 & 2.94 \\
\hline Max & 75.29 & 2.80 & 55.46 & 861.63 & 565.00 & 196.96 & 24.39 & 87.73 & 376.94 \\
\hline
\end{tabular}

CCI - Chlorophyll Content Index; LN - Leaf Number; PH - Plant Height; LA - (Leaf Width x Leaf Length x 0,85); TPB - Total Plant Biomass; FAGW - Fresh Above Ground Weight; DAGW - Dry Above Ground Weight; DCW - Dry Corm Weight; FCW - Fresh Corm Weight. $\mathrm{c}$ - Significant $\mathrm{G} \times \mathrm{E}$ interaction (ANOVA, $\mathrm{p} \leq 0.05$ )

\section{Agronomic traits}

All five parameters, Total Plant Biomass (TPB), Fresh and Dry Above Ground Biomass (FAGW and DAGW) and Fresh and Dry Corm Weight (FCW and DCW), (Tables 3 and 4), were significantly higher under control than under stress $(\mathrm{p} \leq 0.001)$, and all of them differed significantly among cultivars ( $\mathrm{p} \leq 0.001)$. Also, a significant treatment $x$ genotype interaction was detected in all of them $(p \leq 0.001)$, showing that variation on these parameters can discriminate crop behaviour under stress conditions. Mean TPB under stress was on average $34.68 \%$ of control plants. Only two cultivars showed an increase in plant biomass under stress with the largest gain being $20.87 \%$ of the control plants biomass in cultivar 2242. For the remaining 31 cultivars, the highest loss ( $97 \%$ of the control plants biomass) was in cultivar 2237 .
Three of the tested cultivars increased their FAGW, and cultivar 2216 showed the higher FAGW under drought stress. In contrast, cultivar 2237 showed a 95\% loss in relation to control plants. Mean FAGW under stress was on average only $29.74 \%$ of the control plants. Mean DAGW under stress was on average only $35.53 \%$ of control plants. Two of the tested cultivars slightly increased their DAGW, with cultivar 2207 showing the best performance (more 16.27\%). Cultivar 2237 had similar performance in FAGW and DAGW, with a decrease of $94 \%$ of biomass. Mean FCW under stress was on average only $34.93 \%$ of the control. Only three of the tested cultivars showed an increase of yield under stress, with maximal biomass value reaching $151.57 \%$ in cultivar 2234 and maximal biomass loss of $98 \%$ in cultivar 2237. 
70

Dry corm biomass (DCW) was recorded as an important measure of corm quality, in which heavier dry corms translate into an increased content of starch. Mean DCW under stress was on average $34.23 \%$ of control. Three of the tested cultivars increased their dry yield under stress, with cultivar 2056 presenting the best performance of $192.89 \%$ and with cultivar 2237 showing a decrease of $99 \%$ of dry corm biomass.

\section{Stress tolerance indices}

Fresh corm weight as a measure of taro yield was used to calculate harvest (HI), stress susceptibility (SSI) and tolerance (STI) indices (Tables 5 and 6). Mean HI was slightly lower (7.62\%) under stress. There was no statistically significant differences among cultivars, and between treatments for HI. Differences under stress indices are significant among cultivars for SSI and STI ( $\mathrm{p} \leq 0.001)$.

Using corm biomass, WUE and stress tolerance indices, we were able to rank taro cultivars according to their drought tolerance (Table 5). The Madeiran, Canary Islands and Azorean cultivars are among the ten top drought tolerant cultivars as measured by STI (Table 5), and in general also show a better WUE and HI. SPC cultivars dominate in SSI top ranking with some of them showing good WUE.

\section{Multivariate analysis}

Factor analysis (FA), using the Principal Component Analysis (PCA) as extraction method and Varimax rotation, was performed to verify if the assay data variation and obtained factors could explain cultivars performance and identify drought tolerance (Fig. 1). Ten variables, showing significant differences among cultivars and treatments were used in the FA including: plant growth (PH, LA) and agronomic (TPB, FCW, DRW, FAGW, DAGW) traits, WUE and stress indices (SSI, STI). In spite that CCI satisfies both previous conditions, it was not used because it decreased the sample adequacy for the analysis. Result of the Kaiser Meyer Olkin Test (KMO) was 0.749, indicating that sampling was adequate. Two factors were extracted, with eigenvalues $>1$, explaining $81.73 \%$ of total variability, with $1^{\text {st }}$ component representing $63.17 \%$ and the $2^{\text {nd }}, 18.55 \%$ (Fig. 1). Factor 1 appears to be associated with variables for plant growth, agronomic traits and WUE under control, while factor 2 was linked to the same variables for plants under stress. SSI and STI were equally associated with both components. STI scored positively in both components, while SSI scored positively in factor 1 and negatively in factor 2 . Taro cultivars distribution along plot components indicates that these agronomic traits and stress indices can be used to discriminate taro cultivars with regard to drought tolerance (Fig. 1).

Table 4. Data for Harvest Index (HI), Water Use Efficiency (WUE), Stress Susceptibility Index (SSI) and Stress Tolerance Index (STI), obtained under control and drought conditions

\begin{tabular}{|c|c|c|c|c|c|c|}
\hline Accession & $\mathrm{HI}(\%)$ control & HI (\%) drought & WUE $\left(\mathrm{g} \mathrm{m}^{-3}\right)$ control & WUE $\left(\mathrm{g} \mathrm{m}^{-3}\right)$ drought & SSI & STI \\
\hline 2056 & 52.36 & 69.66 & $3,070.52$ & $5,433.89$ & -6.75 & 0.34 \\
\hline 2057 & 58.78 & 60.51 & $5,026.10$ & $1,712.50$ & 0.69 & 0.23 \\
\hline 2058 & 73.04 & 50.91 & $7,397.58$ & $5,450.35$ & 1.10 & 0.93 \\
\hline 2060 & 57.99 & 28.80 & $6,583.49$ & $4,874.03$ & 1.14 & 0.43 \\
\hline 2061 & 62.83 & 68.67 & $8,724.89$ & $9,303.47$ & 0.60 & 2.66 \\
\hline 2062 & 49.78 & 31.75 & $4,185.47$ & $1,399.64$ & 1.39 & 0.07 \\
\hline 2183 & 70.17 & 56.99 & $5,610.12$ & $3,685.16$ & 0.95 & 0.77 \\
\hline 2184 & 66.20 & 34.20 & $5,309.26$ & $1,893.63$ & 1.38 & 0.27 \\
\hline 2186 & 67.15 & 81.35 & $5,034.95$ & $2,469.95$ & 1.12 & 0.43 \\
\hline 2207 & 64.39 & 44.30 & $5,238.47$ & $5,038.70$ & 0.83 & 0.70 \\
\hline 2208 & 75.21 & 32.06 & $6,034.86$ & $1,778.36$ & 1.43 & 0.17 \\
\hline 2209 & 70.66 & 44.61 & $7,795.77$ & $2,025.36$ & 1.34 & 0.38 \\
\hline 2210 & 61.10 & 53.59 & $2,778.52$ & $1,728.96$ & 1.02 & 0.13 \\
\hline 2211 & 48.36 & 49.81 & $15,821.61$ & $4,544.71$ & 1.27 & 1.55 \\
\hline 2212 & 72.97 & 59.73 & $1,814.00$ & $2,453.48$ & 0.73 & 0.16 \\
\hline 2213 & 62.57 & 68.78 & $8,556.76$ & $4,017.78$ & 1.11 & 0.98 \\
\hline 2214 & 66.87 & 68.71 & $5,884.44$ & $4,264.78$ & 0.85 & 0.89 \\
\hline 2215 & 55.04 & 65.82 & $2,159.10$ & $2,288.82$ & 0.18 & 0.15 \\
\hline 2216 & 46.16 & 46.22 & $15,290.68$ & 7426.31 & 1.13 & 1.61 \\
\hline 2232 & 51.59 & 35.60 & $2,690.03$ & 675.12 & 1.12 & 0.02 \\
\hline 2233 & 45.29 & 62.20 & $2,381.80$ & $2,950.22$ & -0.50 & 0.09 \\
\hline 2234 & 29.80 & 70.12 & $1,769.75$ & $3,523.79$ & -8.25 & 0.08 \\
\hline 2235 & 24.90 & 16.56 & $2,610.39$ & 301.88 & 1.01 & 0.00 \\
\hline 2236 & 67.86 & 50.69 & $2,309.53$ & $2,041.82$ & 0.72 & 0.12 \\
\hline 2237 & 35.76 & 21.27 & $4,388.99$ & 49.40 & 1.50 & 0.00 \\
\hline 2238 & 61.85 & 47.99 & $3,105.92$ & 2074.76 & 1.11 & 0.13 \\
\hline 2239 & 49.10 & 56.61 & $3,628.00$ & $4,808.17$ & -0.06 & 0.38 \\
\hline 2240 & 39.07 & 52.04 & $4,955.31$ & $4,347.11$ & 0.03 & 0.28 \\
\hline 2241 & 74.27 & 47.82 & $5,158.84$ & $4,495.31$ & 0.68 & 0.27 \\
\hline 2242 & 49.90 & 77.04 & $1,017.61$ & $2,288.82$ & -0.02 & 0.06 \\
\hline 2244 & 66.74 & 67.38 & $1,760.91$ & $2,815.74$ & -2.38 & 0.16 \\
\hline 2245 & 57.29 & 62.26 & $2,468.81$ & $1,300.84$ & 0.53 & 0.09 \\
\hline 2246 & 46.03 & 53.78 & 433.59 & 510.46 & 0.95 & 0.00 \\
\hline Mean & 57.00 & 52.66 & $4,878.66 \mathrm{abc}$ & $3,150.71 \mathrm{abc}$ & $0.24 \mathrm{a}$ & $0.44 \mathrm{a}$ \\
\hline S.E. & 2.26 & 2.77 & 607.79 & 357.07 & 0.37 & 0.10 \\
\hline Min & 24.90 & 16.56 & 433.59 & 49.40 & -8.25 & 0.00 \\
\hline Max & 75.21 & 81.35 & $15,821.61$ & $9,303.47$ & 1.50 & 2.66 \\
\hline
\end{tabular}

a - Significant differences between accessions (ANOVA, $p \leq 0.05$ )

$\mathrm{b}$ - Significant differences between stress and non stress conditions (ANOVA, $\mathrm{p} \leq 0.05$ )

c - Significant $\mathrm{G} \times \mathrm{E}$ interaction (ANOVA, $\mathrm{p} \leq 0.05$ ) 


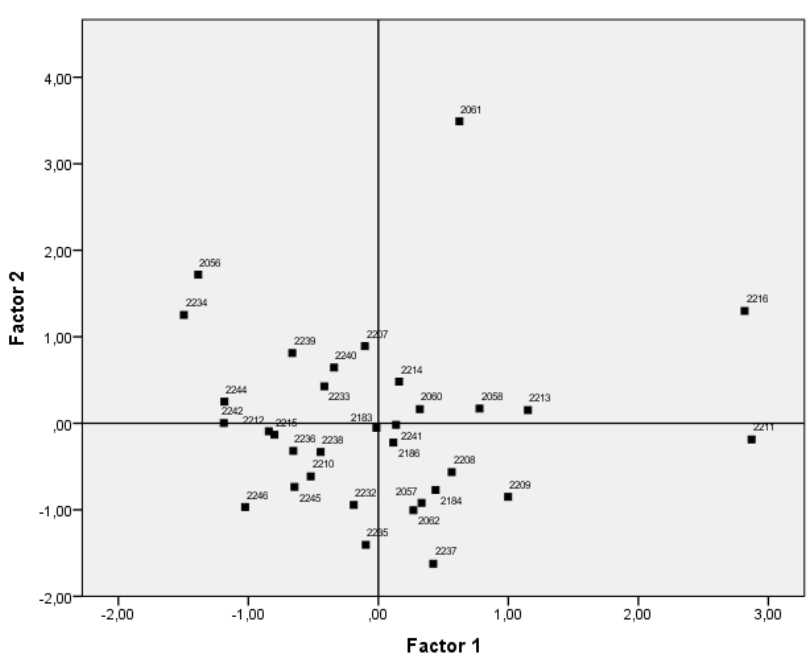

Fig. 1. Factor Analysis, using Principal Component Analysis (PCA) as extraction method, and 10 significant variables to discriminate the behaviour of taro cultivars under drought stress. Factor 1 and 2 explain $63.17 \%$ and $18.55 \%$ of observed variability, respectively

\section{Discussion}

Screening of taro genotypes for drought tolerance is a challenging task, due to the crop growth characteristics, and cultivation under persistent wet conditions. Previous studies were conducted only on a limited number of cultivars, with more than 25 morphological, agronomic and physiological parameters used to assess taro drought tolerance (Sivan, 1995; Bussel and Bonin, 1998; Manyatsi et al., 2011; Mabhaudhi et al., 2013; El-Zohiri and Abd El-Aal, 2014; Mabhaudhi and Modi, 2015). Sivan (1995) calculated that the crop water use

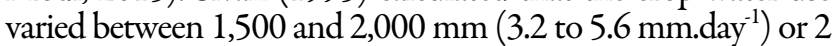
to $6.5 \mathrm{~mm}^{-d a y^{-1}}$ in Florida. Onwueme (1999) showed that optimal yields required 2,500 to 3,000 $\mathrm{mm}$ of water. Fares (2008) used Kc described by Allen et al. (1998) to determine taro water (ETa) needs in 115 to $120 \%$ of local evapotranspiration. Mabhaudhi and Modi (2013) used this approach to define irrigation regimes and modulate crop behaviour under drought stress.

Potential evapotranspiration (ETo) during the drought trial on Madeira, measured at open field, was on average 3.8 $\mathrm{mm}$ day $^{-1}$ (data not shown). A four week pilot trial with daily

Table 5. Taro cultivars, ranked in decreasing order of drought tolerance, using Harvest index (HI), Water Use Efficiency (WUE), Stress Susceptibility Index (SSI) and Stress Tolerance Index (STI)

\begin{tabular}{cccccc}
\hline $\begin{array}{c}\text { HI } \\
(\%) \\
\text { control }\end{array}$ & $\begin{array}{c}\text { HI } \\
(\%) \\
\text { drought }\end{array}$ & $\begin{array}{c}\text { WUE } \\
\left(\mathrm{g} \mathrm{m}^{-3}\right) \\
\text { control }\end{array}$ & $\begin{array}{c}\text { WUE } \\
\left(\mathrm{g} \mathrm{m}^{-3}\right) \\
\text { drought }\end{array}$ & SSI & STI \\
\hline 2208 & 2186 & 2211 & 2061 & 2234 & 2061 \\
\hline 2241 & 2242 & 2216 & 2216 & 2056 & 2216 \\
\hline 2058 & 2234 & 2061 & 2058 & 2244 & 2211 \\
\hline 2212 & 2056 & 2213 & 2056 & 2233 & 2213 \\
\hline 2209 & 2213 & 2209 & 2207 & 2239 & 2058 \\
\hline 2183 & 2214 & 2058 & 2060 & 2242 & 2214 \\
\hline 2236 & 2061 & 2060 & 2239 & 2240 & 2183 \\
\hline 2186 & 2244 & 2208 & 2211 & 2215 & 2207 \\
\hline 2214 & 2215 & 2214 & 2241 & 2245 & 2186 \\
\hline 2244 & 2245 & 2183 & 2240 & 2061 & 2060 \\
\hline 2184 & 2233 & 2184 & 2214 & 2241 & 2209 \\
\hline 2207 & 2057 & 2207 & 2213 & 2057 & 2239 \\
\hline 2061 & 2212 & 2241 & 2183 & 2236 & 2056 \\
\hline 2213 & 2183 & 2186 & 2234 & 2212 & 2240 \\
\hline 2238 & 2239 & 2057 & 2233 & 2207 & 2241 \\
\hline 2210 & 2246 & 2240 & 2244 & 2214 & 2184 \\
\hline 2057 & 2210 & 2237 & 2186 & 2246 & 2057 \\
\hline 2060 & 2240 & 2062 & 2212 & 2183 & 2208 \\
\hline 2245 & 2058 & 2239 & 2242 & 2235 & 2244 \\
\hline 2215 & 2236 & 2238 & 2215 & 2210 & 2212 \\
\hline 2056 & 2211 & 2056 & 2238 & 2058 & 2215 \\
\hline 2232 & 2238 & 2210 & 2236 & 2238 & 2238 \\
\hline 2242 & 2241 & 2232 & 2209 & 2213 & 2210 \\
\hline 2062 & 2216 & 2235 & 2184 & 2232 & 2236 \\
\hline 2239 & 2209 & 2245 & 2208 & 2186 & 2245 \\
\hline 2211 & 2207 & 2233 & 2210 & 2216 & 2233 \\
\hline 2216 & 2232 & 2236 & 2057 & 2060 & 2234 \\
\hline 2246 & 2184 & 2215 & 2062 & 2211 & 2062 \\
\hline 2233 & 2208 & 2212 & 2245 & 2209 & 2242 \\
\hline 2240 & 2062 & 2234 & 2232 & 2184 & 2232 \\
\hline 2237 & 2060 & 2244 & 2246 & 2062 & 2235 \\
\hline 2234 & 2237 & 2242 & 2235 & 2208 & 2246 \\
\hline 2235 & 2235 & 2246 & 2237 & 2237 & 2237 \\
\hline & & & & & \\
\hline
\end{tabular}

Table 6. Pearson's correlations detected between traits, indices and factor 1 and 2 of FA

\begin{tabular}{|c|c|c|c|c|c|c|c|c|c|c|}
\hline & SSI & STI & HIC & HID & $\mathrm{FCW}(\mathrm{g}) \mathrm{C}$ & FCW $(\mathrm{g}) \mathrm{D}$ & TPB (g) C & TPB (g) D & WUE C & WUE D \\
\hline SSI & - & & & & & & & & & \\
\hline STI & n.s. & - & & & & & & & & \\
\hline $\mathrm{HIC}$ & n.s. & n.s. & - & & & & & & & \\
\hline HID & $-0.420^{\circ}$ & n.s. & n.s. & - & & & & & & \\
\hline $\mathrm{FCW}(\mathrm{g}) \mathrm{C}$ & $0.357^{\circ}$ & $0.789^{* *}$ & $0.361^{\circ}$ & n.s. & - & & & & & \\
\hline FCW $(\mathrm{g}) \mathrm{D}$ & n.s. & $0.802^{\prime \prime}$ & n.s. & $0.560^{\circ}$ & $0.450^{\circ}$ & - & & & & \\
\hline TPB (g) C & n.s. & $0.770^{*}$ & n.s. & n.s. & $0.925^{\prime \prime}$ & $0.433^{\circ}$ & - & & & \\
\hline TPB (g) D & n.s. & $0.828^{\prime \prime}$ & n.s. & n.s. & $0.580^{\circ}$ & $0.921 "$ & $0.583^{\circ}$ & - & & \\
\hline WUE C & n.s. & 0.772 & n.s. & n.s. & $0.926^{\prime \prime}$ & $0.427^{\circ}$ & $0.998^{\prime \prime}$ & $0.581^{*}$ & 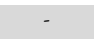 & \\
\hline WUE D & n.s. & 0.826 & n.s. & n.s. & $0.581^{\prime \prime}$ & $0.920 "$ & $0.582^{\prime \prime}$ & $0.999^{\circ}$ & $0.580^{\prime \prime}$ & - \\
\hline Factor 1 & $0.510^{*}$ & $0.637^{\prime \prime}$ & n.s. & n.s. & $0.884 "$ & n.s. & $0.948^{*}$ & $0.348^{*}$ & $0.947^{\prime \prime}$ & $0.346^{\circ}$ \\
\hline Factor 2 & $-0.449^{\circ}$ & $0.675^{\prime \prime}$ & n.s. & $0.505^{\prime \prime}$ & n.s. & $0.943^{\prime \prime}$ & n.s. & $0.900^{\circ}$ & n.s. & $0.900^{\circ}$ \\
\hline
\end{tabular}

SSI - Stress Susceptibility Index; STI - Stress Tolerance Index; HI - Harvest Index; FCW - Fresh Corm Weight; TPB - Total Plant Biomass; WUE - Water Use Efficiency. C - Control; D - Drought;

n.s. - not significant; ${ }^{*}$ significant at $\mathrm{p} \leq 0.05 ;^{* *}$ significant at $\mathrm{p} \leq 0.001$ 
72

monitoring of adult plants evapotranspiration in the assay greenhouses, determined a water use of $10.7 \pm 3.5 \mathrm{~mm}^{-1 a y}{ }^{-1}$, and showed that $120 \%$ of ETo $\left(4.03 \mathrm{~mm} \mathrm{day}^{-1}\right)$ was not enough to maintain plants fully hydrated in the test conditions. Based on these findings, the irrigation regimes for the assay were based in measured ET.

In the present study, 11 traits and four derived variables were used to discriminate plants tolerance to drought, following the methodology developed by Ganança et al. (2015). These parameters were selected based on their connection with known strategies to avoid or answer to drought (Langridge and Reynolds, 2015), namely plant effective growth, WUE, nutrients allocation and yield. Drought tolerance was defined as yield stability under stress conditions, and a small number of traits at harvest were used for this assessment, with yield potential defined as the maximum yield obtained under non-stress conditions. Drought resistance is determined by dehydration avoidance or tolerance. Dehydration-avoidant phenotypes normally have smaller plants and leaf area, and are associated with low yield potential (Blum, 2005), and therefore, are not interesting from breeders perspective. Genotypes showing high yield both in stressed and non-stressed conditions were considered drought tolerants (Ganjeali et al., 2011; Farshadfar et al., 2013).

As expected, in the present work all the plant growth traits were reduced under drought, with variance analysis showing significant differences among cultivars, and significant influence of water scarcity in plant development. Traits decrease can be a result of the reduction of nutrients uptake and assimilation, increased water transpiration or energy allocation to face the stress. Reduction of LA is a known drought avoidance strategy used by taro to cope with low water availability, and Mabhaudhi and Modi (2015) associated the reduction of leaf number to a drought escape mechanism involving leaf shedding, phenological plasticity and a shortened crop cycle. The present data reveals different responses among cultivars, suggesting that both avoidance and tolerance strategies could be used by taro. For instance, the three top cultivars (2061, 2216 and 2211) for potential yield are representative of the different approaches used to cope with drought stress. Cultivar 2061 reduced leaf number, but maintained plant height and leaf area, while 2211 and 2216 maintained leaf number, but decreased plant height and leaf area. In 2061, LA was already lower under control conditions, showing potential for lower water loss and transpiration, than the other cultivars. Cultivars bred for water-limited environments normally have a constitutively reduced leaf area. However, some species, such as sorghum, maintain high LA, discarding older leaves and maintaining turgor in younger leafs (Blum, 2005). Similarly, drought tolerant aroids also maintain high leaf water content, reducing LN or LA in less extent than the sensitive ones (Sivan, 1995).

In our work, only one third of the tested cultivars decreased CCI to avoid drought. Leaf chlorophyll content has been shown to decrease with drought stress (Mabhaudhi et al., 2013; Mabhaudhi and Modi, 2015), suggesting that the decrease in chlorophyll content could be a plant strategy to dissipate radiation energy and down regulate photosynthesis, due to the $\mathrm{CO}_{2}$ scarcity resulting from stomatal closure. The majority of cultivars included in this study did not decrease CCI to compensate drought, and no significant correlations between $\mathrm{CCI}$ and other stress indices were observed.
WUE is generally considered a measure of drought resistance. Plants showing higher WUE are considered normally drought tolerant (Blum, 2005). WUE normally decreases under stress conditions, but Mabhaudhi et al. (2013) could not find differences in taro cultivars grown under control and stress conditions. However, they found that more tolerant cultivars had higher WUE. Bussel and Bonin (1998) also reported that a tolerant variety had higher WUE. In our work, cultivars showing higher WUE under full watering conditions had higher TPB and yield potential, while the ones that increase or maintain WUE under drought improve or show a small decrease in TPB and yield. Results show that WUE is a essential parameter for discriminating tolerance in taro.

Concerning the agronomic and yield traits, plant biomass, including TPB, FAGW and FCW were also significantly reduced under drought (Tables 3, 4, 5). Our results show that TPB appears highly correlated ( $\mathrm{p} \leq 0.001)$ with corm biomass, both under control or under drought conditions (Table 6). Plants that were able to maintain TPB under drought were also capable to develop and maintain CW, which is also highly correlated with PH and LA (data not shown). These traits alleviate negative influences of fixation of nutrients and allocation of biomass in the corm (CW). Some cultivars (2056, 2234 and 2242), presenting lower yield potential, were able to maintain or slightly increase corm biomass under drought. These cultivars can be classified as expressing drought avoidance mechanisms (Ganjeali et al., 2011; Farshadfar et al., 2013), and efficiently transferred resources to sustain corm formation. However, these results need to be carefully interpreted, as they presented very low yield potential under control conditions and should be highly sensitive to other external variants. Cultivars 2061, 2216 and 2211 showed high potential yield, but suffered a yield reduction under drought. Nevertheless, cultivar 2061 is more efficient in biomass accumulation than cultivars 2211 and 2216. In conclusion, cultivars with high potential yield, heavily reduce canopy size and biomass, leading to decrease of yield under drought. Better performers minimised biomass and canopy loss. Mabhaudhi and Modi (2015) and Mabhaudhi et al. (2013) attributed lower yield in all studied taro cultivars to plant energetic investment towards drought avoidance mechanisms, reducing growth and biomass accumulation. In our study, some cultivars showed TPB increase or low reduction, showing that they can avoid or tolerate drought and efficiently transfer resources to corm formation in detriment of canopy formation. HI was not statistically affected by drought stress and appears to be correlated with corm biomass ( $\mathrm{p} \leq 0.05)$, but not with TPB or WUE. However, HI was slightly lower under stress conditions, which remains in agreement with previously reported data (Bussel and Bonin, 1998; Mabhaudhi and Modi, 2015). Mabhaudhi and Modi (2015) found that contribution of corm yield to HI under drought was minimal.

In order to classify cultivars for their drought tolerance, yield stability was expressed as STI and SSI (Ganjeali et al., 2011; Farshadfar et al., 2013). STI identifies cultivars with the best yield stability, hence it is one of the best criterions to identify tolerant genotypes (Farshadfar et al., 2013). STI is highly correlated $(\mathrm{p} \leq 0.001)$ with corm biomass under both conditions (Table 6), confirming that it can be used for ranking purposes. On the other hand, SSI reflects the difference between yield under stress and non stress conditions (Table 4). The higher SSI values, the most susceptible is the cultivar. SSI 
had lower, although significant correlations with $\mathrm{CW}$ under control ( $\mathrm{p} \leq 0.001)$, and with HI ( $\mathrm{p}$ 0.05) under drought (Table 6). Consequently, SSI can identify cultivars lacking or having less successful strategies to avoid drought (Ganjeali et al., 2011). Both indices should be considered for ranking cultivars for tolerance, but SSI should be used when taking in account potential yields (Farshadfar et al., 2013). According to STI, cultivars 2061,2216,2211,2213, 2058 and 2214 have the highest yield potential under drought stress. Cultivars 2234, 2056, 2244, 2233, 2239 and 2242, according to SSI, presented a efficient response to drought. Cultivar 2061 showed the highest STI with only slightly decreased yield under stress. On the contrary, cultivars 2234 and 2056 have medium and low STI (low yield potential) but showed resistance, ranking $1^{\text {st }}$ and $2^{\text {nd }}$ in SSI indexes. Among yield tolerant cultivars, 2061 was able to sustain WUE under stress, while 2216 and 2211 were less successful. Cultivars 2234 and 2056 increased their WUE under stress conditions, but their yields were average or low. STI was highly correlated with WUE ( $\mathrm{p} \leq 0.001)$, but not with SSI (Table 6), making it a less appropriate trait for ranking cultivars. Our results confirm that WUE is an important trait in taro drought tolerance, but it is not the only determining factor.

Ploidy data were obtained for 12 of the studied cultivars (Table 1) (Kreike et al., 2004; Traore, 2013). A correlation was found between STI and available ploidy levels (data not shown), indicating that in taro triploids can in fact have an advantage in yield and yield stability under drought. However, no correlation was found with SSI, indicating that with the limited set of data we cannot claim that ploidy levels contribute to drought tolerance.

To confirm ranking of taro cultivars for drought tolerance, we developed a model summarizing the traits variation under stress and non stress conditions, using multivariate statistics. Factorial Analysis (Fig. 1) allowed us to resume the information obtained along two factors, useful to discriminate cultivars regarding drought resistance and yield stability. Factor 1 is strongly correlated with cultivar's behaviour (yield potential) under non stress conditions, while factor 2 is strongly correlated with cultivars behaviour (WUE and resistance) under stress (Table 6). According to Ganjeali et al. (2011), cultivars can be distinguished for their drought resistance by high or low scores for factor 2 . STI was positevely correlated with both factors $(\mathrm{p} \leq 0.001)$, confirming a good association with potential yield and drought tolerance (Tables 6). SSI was positively correlated with factor 1 and negatively correlated with factor $2(p \leq 0.001)$, showing that cultivars with low SSI generally show low yield potential but high drought resistance (Table 6). Cultivars of interest showing both medium-high yield potential and drought tolerance have scores higher than 0 for both factors. Cultivars 2061 and 2216 stand out in this classification. Cultivars 2056 and 2234 excel as having good WUE and drought resistance, but showed low yield. Cultivars with scores below 0 for both factors show very low yield potential and are drought susceptible, standing out cultivars such as 2246, 2245 and 2235. Cultivars 2061 and 2216 probably are good candidates for breeding programs targeting crop improvement or as standards for screening for drought tolerance. In contrast, cultivars 2056 and 2234 can be adapted to drought but with overall low yield potential, are less attractive to taro breeders.
In our work, the Macaronesian cultivars were screened regarding drought for first time. Most of the local cultivars (e.a. cultivars 2061 and 2216) showed higher yield potential, but also STI, WUE and HI, when compared with SPC cultivars. At the same time, SPC cultivars 2233, 2234, 2239 and 2240 showed some drought resistance based on WUE, which in our test was not reflected in yield, but demonstrates existence of stress tolerance strategies. Cultivars presenting different strategies to cope with drought could be used as benchmarks for screening other taro cultivars for drought tolerance, as source of genetic variability for breeding programs, or for studying the molecular and biochemical background of taro drought stress and tolerance.

\section{Conclusions}

In order to screen and classify a relatively high number of taro cultivars, crop water requirements were analysed, and a model of drought conditions was defined, using a plant-soil system to monitor water use. Frequently used traits to assess the crop behaviour under drought conditions were assessed for their discriminating efficiency. A methodology aiming at creating long term stress and screening cultivars based on a small number of parameters collected at harvest was defined. Significant differences in behaviour between cultivars and treatments were observed. Our results confirm that different taro cultivars have different drought avoidance and tolerance strategies to cope with water scarcity. Cultivars with high potential yield, heavily reduce canopy size and biomass, leading to decrease of yield under drought. Better performers minimised biomass and canopy loss. Drought physiological tolerance was observed in cultivars that presented low potential yield, but efficiently transferred resources to enhance corm formation. Cultivars sustaining or increasing WUE under drought were more tolerant. Drought tolerant and susceptible cultivars were classified based on STI and SSI, respectively. Factorial analysis contributed to discriminate cultivars regarding to their drought tolerance and yield stability, confirming cultivars 2061 and 2216 as the most interesting ones, and the physiological tolerance, based on WUE, of cultivars 2056 and 2234. A group of cultivars that show a compromise between yield potential and tolerance, using WUE or metabolism bioenergetics to face drought stress was also identified, namely cultivars $2207,2214,2233$, 2239 and 2240. The cultivars can now be used as benchmarks for screening other taro cultivars for drought tolerance, as source of genetic variability for breeding programs or to study mechanisms involved in plant adaptation to drought.

\section{Acknowledgements}

This work was funded by the European Commission through the project Europe Aid/28-500/C/ACT/TPS, DCI-Food/2009/45. The authors also wish to thank all the members of INEA network that contributed to this work. We also want to thank Dr. George Soteriou, from ARI in Cyprus, Dr. Neftali Castro Martin from the Cabildo de La Palma in Canary Islands, and Dr. Conceição Filipe from FRUTER on the Azores Islands for providing plant material for this study. 


\section{References}

Allen RG, Pereira LS, Raes D, Smith M (1998). Crop evapotranspiration Guidelines for computing crop water requirements. FAO Irrigation and drainage paper 56, Rome:FAO.

Blum A (2005). Drought resistance, water-use efficiency, and yield potentialare they compatible, dissonant, or mutually exclusive? Australian Journal of Agricultural Research 56:1159-1168.

Bussell WT, Bonin MJ (1998). Effects of high and low watering levels on growth and development of taro. New Zealand Journal of Crop and Horticultural Science 26:313-317.

Dwyer LM, Stewart DW, Balchin D (1987). Accurately monitoring and maintaining soil water in greenhouse containers. Canadian Agriculture Engineering 29:89-91.

El-Zohiri SSM, Abd El-Aal AMH (2014). Improve the adverse impacts of water stress on growth, yield and its quality of taro plants by using gycinebetaine, $\mathrm{MgCO}_{3}$ and defoliation under delta conditions. Middle East Journal of Agriculture Research 3(4):799-814.

FAOSTAT(2013).FAOStatistical Database. http://faostat.fao.org.

Fares A (2008). Water management software to estimate crop irrigation requirements for consumptive use permitting in Hawaii. Final Report, University of Hawaìi at Manoa. http://hawaii.gov/dlnr/ cwrm/publishedreports/ PR200808.pdf.

Farshadfar E, Shahandi MA, Romena MH, Hasanalideh AH (2013). Screening of drought tolerance in landraces of bread wheat using agronomic, physiologic and metabolic indicators. Advanced Crop Science 3(5):334349.

Fernandez GCJ (1992). Effective selection criteria for assessing stress tolerance. In: Kuo CG (Ed). Proceedings of the International Symposium on Adaptation of Vegetables and Other Food Crops in Temperature and Water Stress. Tainan AVRDC Publication pp 257270

Fisher RA, Maurer R (1978). Drought resistance in spring wheat cultivars. I. Grain yield responses. Australian Journal of Agricultural Research 29:897-912.

Ganança JFT, Freitas JGF, Nóbrega HGM, Rodrigues V, Antunes G, ... Lebot V (2015). Screening of elite and local taro (Colocasia esculenta) cultivars for drought tolerance. Procedia Environmental Sciences 29:4142.

Ganjeali A, Porsa H, Bagheri A (2011). Assessment of Iranian chickpea (Cicer arietinum L.) germplasms for drought tolerance. Agricultural Water Management 98:1477-1484.
Kreike CM, Van Eck HJ, Lebot V (2004). Genetic diversity of taro, Colocasia esculenta (L.) Schott, in Southeast Asia and the Pacific. Theoretical and Applied Genetics 109:761-768.

Langridge P, Reynolds MP (2015). Genomic tools to assist breeding for drought tolerance. Current Opinion in Biotechnology 32:130-135.

Mabhaudhi T, Modi AT (2015). Drought tolerance of selected South African taro (Colocasia esculenta L. Schott) Landraces. Experimental Agriculture 51:451-466.

Mabhaudhi T, Modi AT, Beletse YG (2014). Parameterisation and evaluation of the FAO-AquaCrop model for a South African taro (Colocasia esculenta L. Schott) landrace. Agricultural and Forest Meteorology 192-193:132-139.

Mabhaudhi T, Modi AT, Beletse YG (2013). Response of taro (Colocasia esculenta L. Schott) landraces to varying water regimes under a rainshelter. Agricultural Water Management 121:102-112.

Manyatsi AM, Mhazo N, Mkhatshwa M, Masarirambi MT (2011). Effect of different in-situ water conservation tillage methods on growth and development of taro (Colocasia esculenta L.). Asian Journal of Agricultural Sciences 3:11-18.

Onwueme I (1999). Taro Cultivation in Asia and the Pacific. Rap Publication. 1999/16. Bangkok. FAO.

Rao VR, Hunter D, Eyzaguirre PB, Matthews PJ (2010). Ethnobotany and global diversity of taro. In: Rao VR et al. (Eds). The Global Diversity of Taro: Ethnobotany and Conservation. Bioversity International, Rome pp 1-5.

Sivan P (1995). Drought tolerance and the effect of potassium supply on growth of taro (Colocasia esculenta (L.) Schott) and tannia (Xanthosoma sagittifolium (L.) Schott).PhD Thesis, University of Queensland.

Traore RE (2013). Étude de la diversité du Taro (Colocasia esculenta (L.) Schott): Cas d'une collection du Burkina Faso et d'une collection internationale [Study of Taro (Colocasia esculenta (L.) Schott) diversity: The case of a Burkina Faso collection and an international collection]. $\mathrm{PhD}$ Thesis, Universitéde Ouagadougou.

Wairiu M, Murari LM, Lese V (2012). Climate Change Implications for Crop Production in Pacific Islands Region. In: Aladjadjiyan A (Ed). Food Production - Approaches, Challenges and Tasks. InTech, Shangai pp 65-86. 